\title{
$\phi$-POSTNIKOV SYSTEMS AND EXTENSIONS OF H-SPACES
}

\section{ALEERT O. SHAR}

ABSTRACT. Let $f: X \rightarrow Y$ be a map of CW complexes and let $\pi: F_{f} \rightarrow X$ be the fibration induced by $f$.

The following theorems are proven:

Theorem. Assume $F\left(=F_{f}\right)$ and $\Omega Y$ are simply connected and that

(a) $f^{*}: H^{n}\left(Y ; \pi_{n}(Y)\right) \rightarrow H^{n}\left(X ; \pi_{n}(Y)\right)$ is epic for all $n$,

(b) $(i \wedge i)^{*}: H^{n}\left(F \wedge F\right.$; coker $\left.f_{n^{*}}\right) \rightarrow H^{n}\left(\Omega Y \wedge \Omega Y\right.$; coker $\left.f_{n *}\right)$ is monic for all $n$ (where $\left.f_{n *}: \pi_{n}(X) \rightarrow \pi_{n}(Y)\right)$.

If $X$ is an H-space then $F$ is an $H$-space such that $\pi: F \rightarrow X$ is an H-map.

Theorem. Assume $Y$ is $(p-1)$-connected, $F$ is $(q-1)$-connected $(p-1 \geq 2, q-1 \geq 1)$ of dimension $<\min (2 p-1, p+q-1)$, and $H^{*}(Y)$ is free. If $X$ is an $H$-space and $f^{*}: H^{n}(Y) \rightarrow H^{n}(X)$ is onto for all $n$ then $F$ is an $H$-space and the map $\pi: F \rightarrow X$ is an $H$-map.

Analogous theorems are shown to hold for loop spaces.

Let $f: X \rightarrow Y$ be a (based) map of CW spaces and let $\Omega Y \stackrel{i}{\rightarrow} F_{f} \stackrel{\pi}{\rightarrow} X$ be the fibration induced by $f$. That is, $F_{f}=\{(x, \eta) \in X \times P Y \mid f(x)=\eta(1)\}$ and $\pi(x, \eta)=x$.

In this paper we will develop a Postnikov-type decomposition which will allow us to prove

Theorem 0.1. Assume $F\left(=F_{f}\right)$ and $\Omega Y$ are simply connected and that

(a) $f^{*}: H^{n}\left(Y ; \pi_{n}(Y)\right) \rightarrow H^{n}\left(X ; \pi_{n}(Y)\right)$ is epic for all $n$,

(b) $(i \wedge i)^{*}: H^{n}\left(F \wedge F\right.$; coker $\left.f_{n *}\right) \rightarrow H^{n}\left(\Omega Y \wedge \Omega Y\right.$; coker $\left.f_{n *}\right)$ is monic for all $n$ (where $f_{n *}: \pi_{n}(X) \rightarrow \pi_{n}(Y)$ ).

If $X$ is an H-space, then $F$ is an $H$-space such that $\pi: F \rightarrow X$ is an H-map.

By adding some stability criteria to Theorem 0.1 we prove

Theorem 0.2. Assume $Y$ is $(p-1)$-connected, $F$ is $(q-1)$-connected $(p-1 \geq 2, q-1 \geq 1)$ of dimension $<\min (2 p-1, p+q-1)$, and $H^{*}(Y)$ is free. If $X$ is an H-space and $f^{*}: H^{n}(Y) \rightarrow H^{n}(X)$ is onto for all $n$ then $F$ 1974.

Received by the editors September 10, 1973 and, in revised form, February 26,

AMS (MOS) subject classifications (1970). Primary 55G45; Secondary 55D45.

Key words and phrases. Postnikov system, $H$-space, $H$-map, obstruction. 
is an H-space and the map $\pi: F \rightarrow X$ is an $H$-map. $\left(H^{*}()\right.$ is cohomology with integer coefficients.)

Theorem 0.3. Let $X$ be a loop space $(X(n-1)$-connected $)$. If the induced maps $f_{*}: \pi_{q}(X) \rightarrow \pi_{q}(Y)$ and $f^{*}: H^{q}\left(Y ; \pi_{q}(Y)\right) \rightarrow H^{q}\left(X ; \pi_{q}(Y)\right)$ are onto for all $q$ and all $q>n$ respectively, then $F$ is a loop space and the map $\pi: F \rightarrow X$ is a loop map.

Theorem 0.4. Assume $Y$ is $(p-1)$-connected, $F$ is $(q-1)$-connected $(p-1 \geq 2, q-1 \geq 1)$ of dimension $<\min (2 p-1, p+q-1)$, and $H^{*}(Y)$ is free. If $X$ is a loop space and $f^{*}: H^{n}(Y) \rightarrow H^{n}(X)$ is onto for all $n$ then $F$ is a loop space and $\pi: F \rightarrow X$ is a loop map.

Let $(X, m)$ and $(Y, n)$ be $H$-spaces. Following Stasheff [2], given $f$ : $X \rightarrow Y$ an $H$-map (i.e., $n(f \times f)$ is homotopic to $f m$ ), we define $X$ an $f-s u b$ $H$-space of $Y$ if $f$ is of the homotopy type of the inclusion of the fibre $F$ into the total space $E$ of a fibration over a space $B$.

It is easy to see that this is equivalent to the following:

Replace $f: X \rightarrow Y$ by the fibration $F{ }_{f} \stackrel{i}{\rightarrow} P_{f} \stackrel{p}{\rightarrow} Y$, with $X \simeq P_{f}$ and $p$ equivalent to $f$.

Then $P_{f} \rightarrow Y$ is induced by a map $Y \rightarrow B$.

If $X$ and $F_{f}$ are loop spaces and $\pi: F_{f} \rightarrow X$ is a loop map, we can extend the fibration sequence $F_{f} \rightarrow X \rightarrow Y$ to $F_{f} \rightarrow X \rightarrow Y \rightarrow B_{F} \rightarrow B_{X}$ so that as a corollary of Theorems 0.3 or 0.4 we get

Theorem 0.5. Let $f:(X, m) \rightarrow(Y, n)$ be an $H$-map with $X$ a loop space and $m$ the loop multiplication. If the conditions of Theorems 0.3 or 0.4 are satisfied, then $X$ is an f-sub-H-space of $Y$.

In $\$ 1$ we define and develop a $\phi$-Postnikov system and prove the general results needed for this paper. $\$ 2$ contains a proof of a proposition which give conditions which are internal to the $\phi$-Postnikov construction that insures $F$ is an $H$-space. In $\$ 3$, we prove a theorem which immediately specializes to Theorem 0.1, and in $\$ 4$, we prove Theorem 0.2. In $\$ 5$ we will make the obvious generalization to loop spaces. I would like to thank Professor Arthur Copeland, Jr. for his suggestions and comments.

1. $\phi$-Postnikov systems. Let $\phi: Z^{+} \rightarrow Z^{+}$be a nondecreasing function from the nonnegative integers onto themselves and let $X$ be a connected simple CW complex with $\pi_{1}(X)$ abelian. We define a $\phi$-Postnikov system for $X$ as follows:

Definition 1.1. A $\phi$-Postnikov system $\left\{X_{n}, \pi_{n}, p_{n}, q_{n}, k^{n}\right\}$ for $X$ consists 
of a family of spaces $X_{n}\left(X_{0}=*\right)$ and abelian groups $\pi_{n}, n \in Z^{+}$with maps $p_{n}: X_{n} \rightarrow X_{n-1}, q_{n}: X \rightarrow X_{n}$, and $k^{n}: X_{n} \rightarrow K\left(\pi_{n}, \phi(n)+2\right)$ such that

(1) $q_{n-1}=p_{n} q_{n}$,

(2) $p_{n}: X_{n} \rightarrow X_{n-1}$ is equivalent to the fibration induced by $k^{n}$,

(3) $\left(q_{n}\right)_{*}: \pi_{i}(X) \rightarrow \pi_{i}\left(X_{n}\right)$ is an. isomorphism for $i \leq \phi(n) ; \pi_{i}\left(X_{n}\right)=0$, $i>\phi(n)+1$.

The following are examples of $\phi$-Postnikov systems.

Example 1.2. Let $\left\{X_{n}, \pi_{n}, p_{n}, q_{n}, k^{n}\right\}$ be the standard Postnikov system for $X$, i.e., $\phi=\mathrm{Id}, \pi_{n}=\pi_{n}(x)$ and $k^{n}$ a representative of the transgression of the fundamental class of the fibre of $q_{n-1}: X \rightarrow X_{n-1}$ (e.g., [1] or [4]).

Example 1.3. Let $X$ be a space and let $f_{n}: \pi_{n}(x) \rightarrow \pi_{n}^{\prime}$ be an epimorphism for each $n$. Let $\phi(n)=[n / 2]([s]$, the greatest integer less than or equal to $s)$ and let $f_{n c}: K\left(\pi_{n+1}(x), n+2\right) \rightarrow K\left(\pi_{n+1}, n+2\right)$ be a realization of $f_{n}$. We then may construct the $\phi$-Postnikov system $\left\{X_{n}, \pi_{n}, p_{n}, q_{n}, k^{n}\right\}$ (with $\pi_{2 n}=\pi_{n+1}^{\prime}, \pi_{2 n+1}=\operatorname{ker} f_{n}$ ) inductively as follows:

Assume we have constructed

$$
X_{2 n} \stackrel{p_{2 n}}{\longrightarrow} X_{2 n-1} \rightarrow \cdots \rightarrow X_{0}=* \quad \text { with } q_{2 n}: X \rightarrow X_{2 n}
$$

inducing isomorphisms in homotopy in dimensions $\leq \phi(2 n)=n$ and $\pi_{i}\left(x_{2 n}\right)=$ 0 for $i>n$. Let $k^{2 n}: X_{2 n} \rightarrow K\left(\pi_{n+1}^{\prime}, n+2\right)$ be the composition $f_{(n+1) i} \circ k^{\prime n}$, where $k^{\prime n}$ is constructed as in Example 1.2, and let $X_{2 n+1}$ be the total space of the fibration induced by $k^{2 n}$. It is easy to see that $q_{2 n+1}$ and $p_{2 n+1}$ can be constructed satisfying the conditions of Definition 1.1.

If we let $F_{n}$ be the fibre of $q_{2 n+1}: X \rightarrow X_{2 n+1}$ we see that $\pi_{n+1}\left(F_{n}\right)=$ ker $f_{n}$. Let $k^{2 n+1}: X_{2 n+1} \rightarrow K\left(\pi_{n+1}\left(F_{n}\right), n+2\right)$ be a representative of the transgression of the fundamental class. $X_{2 n+2}$ may now be constructed with the desired properties.

Example 1.4. Let $f: X \rightarrow Y$. Then by a suitable refinement of Example 1.3 we may construct $\phi$-Postnikov systems $\left\{X_{n}, \pi_{n}^{X}, p_{n}^{X}, q_{n}^{X}, k_{X}^{n}\right\}$ and $\left\{Y_{n}, \pi_{n}^{Y}, p_{n}^{Y}, q_{n}^{Y}, k_{Y}^{n}\right\}$ for $X$ and $Y$ respectively, such that $\pi_{2 n}^{X}=\operatorname{im} f_{n+1 *}$, $\pi_{2 n+1}^{X}=\operatorname{ker} f_{n+1 *}\left(f_{n *}: \pi_{n}(X) \rightarrow \pi_{n}(Y)\right), \pi_{2 n}^{Y}=\pi_{n+1}(Y)$ and $\pi_{2 n+1}^{Y}=0$.

Further, we may construct these spaces and maps (see [1]) to yield the following commutative diagrams:

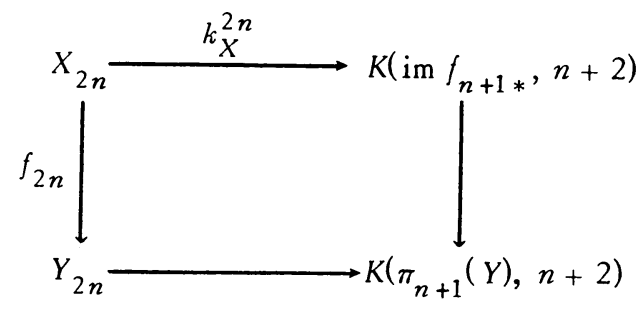




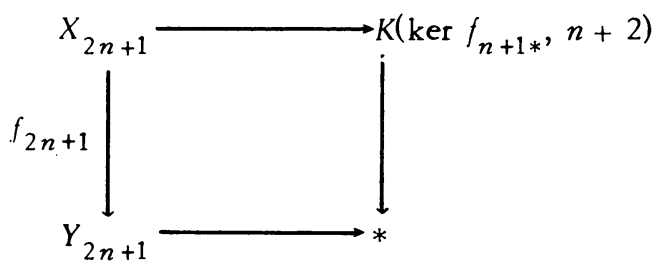

$f_{m}: X_{m} \rightarrow Y_{m}$ is induced from $f_{m-1}$ by considering $X_{m}$ and $Y_{m}$ as the total spaces of the fibrations induced by $k_{X}^{m-1}$ and $k_{Y}^{m-1}$ respectively, with the first nontrivial $f_{m}$ the realization of the coefficient homomorphism $f_{*}$ in homotopy.

In much of what follows, we will need homotopy commutativity of

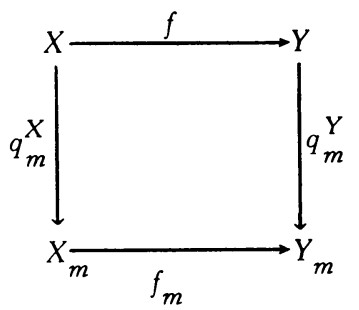

To do this, we need the following lemma.

Lemma 1.6. If $X$ is $(r-1)$-connected and $Y$ is $(s-1)$-connected and $f^{*}: H^{q}\left(Y ; \pi_{q}(Y)\right) \rightarrow H^{q}\left(X ; \pi_{q}(Y)\right)$ is onto for $q>\max (r+1, s+1)$, then there exist $\phi$-Postnikov systems for $X$ and $Y$ such that (1.5) homotopy commutes.

Proof. We use an inductive argument. Assume $f_{m} q_{m}^{X} \sim q_{m}^{Y}$ f. If $m=2 n+1$, $Y_{2 n+2}=Y_{2 n+1}$ and $f_{2 n+2}$ may be defined by $f_{2 n+1} p_{2 n+2}^{X} q_{2 n+2}^{X}$.

If $m=2 n$ we have that $Y_{2 n+1} \rightarrow Y_{2 n}$ is a principal fibration of type $\left(\pi_{n+1}(Y), n+2\right)$. Since $p^{Y} f_{2 n+1} q_{2 n+1}^{X} \sim p^{Y} q_{2 n+1}^{Y} f$, there is an element

$$
\tau \in\left[X, K\left(\pi_{n+1}(Y), n+1\right)\right]=H^{n+1}\left(X ; \pi_{n+1}(Y)\right)
$$

such that $\psi\left(\tau, q_{2 n+1}^{Y} f\right)=f_{2 n+1} q_{2 n+1}^{X}$ (see [4]), where $\psi$ is the action of the fibre of $Y_{2 n+1} \rightarrow Y_{2 n}$ on $Y_{2 n+1}$.

If $f^{*}$ is onto take $\tau^{\prime} \in\left[Y ; K\left(\pi_{n+1}(Y), n+1\right)\right]=H^{n+1}\left(Y ; \pi_{n+1}(Y)\right)$ in $f^{*-1}(\tau)$ and change the lifting $q_{2 n+1}^{Y}$ of $q_{2 n}^{Y}$ to the lifting $\psi\left(\tau^{\prime}, q_{2 n+1}^{Y}\right)^{n+1}=q_{2 n+1}^{\prime}$. By the naturality of the action $\psi$ we get the required result.

In all that follows we will assume that $f: X \rightarrow Y$ satisfies the hypothesis of Lemma 1.6 and call that condition Obl.

If we define $F_{m}$ as the total space of the fibration induced by $f_{m}$ we get 
a $\phi$-Postnikov system for $F(\phi=[n-1 / 2]),\left\{F_{n}, \pi_{n}^{F}, p_{n}^{F}, q_{n}^{F}, k_{F}^{n}\right\}$ with $\pi_{2 n}^{F}=$ coker $f_{n+1 *}, \pi_{2 n+1}^{F}=\operatorname{ker} f_{n+1 *}$ and such that the following diagrams commute:
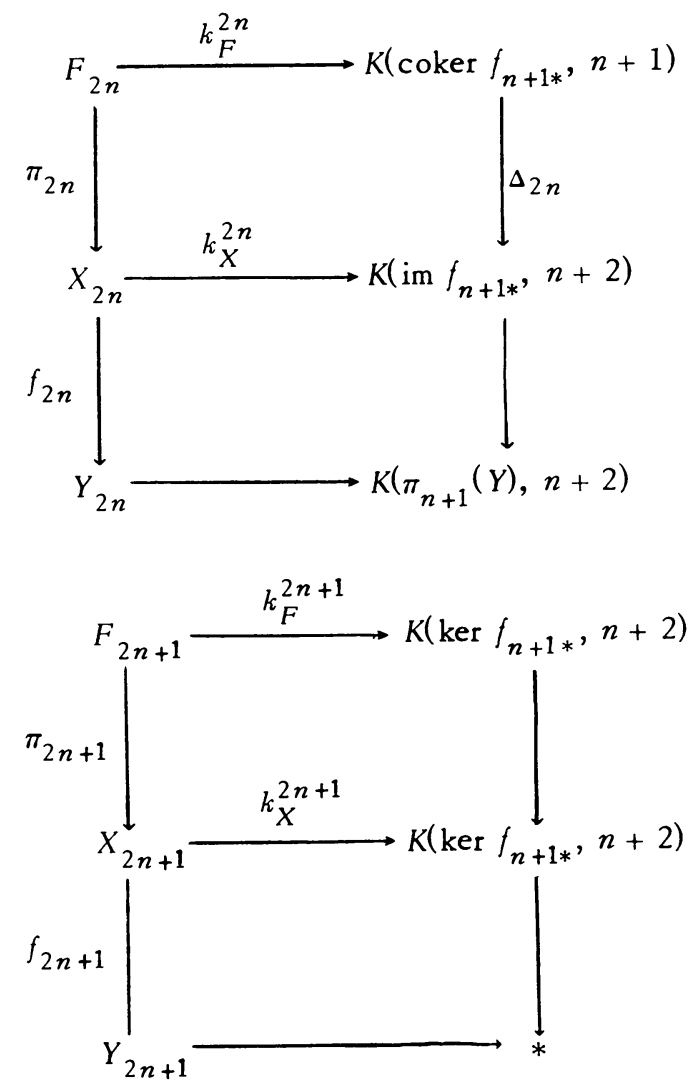

The maps $\pi$ and $p_{m}^{F}: F_{m} \rightarrow F_{m-1}$ are determined by considering $F_{m}$ as the total space of the fibration induced by $k_{F}^{m-1}$.

If $X$ is an $H$-space ( $\Omega$ space), then the spaces $X_{m}$ are $H$-spaces $(\Omega$ spaces) and the maps $q_{m}^{X}, p_{m}^{X}$, and $k_{X}^{m}$ are $H$-maps. If each $F_{m}$ is an $H$ space ( $\Omega$ space) and each $k_{F}^{m}$ is an $H$-map $(\Omega$-map) then $F$ is an $H$-space ( $\Omega$-space), and if the maps $\pi_{m}: F_{m} \rightarrow X_{m}$ are $H$-maps ( $\Omega$-maps) then so is $\pi: F \rightarrow X$ (see Kahn [1], Stasheff [2], [3]).

2. Internal techniques. To determine conditions under which $F$ is an $H$-space the following two technical lemmas are useful.

Lemma 2.1. If $F_{2 n-1}$ and $X_{2 n-1}$ are $H$-spaces and the maps $\pi_{2 n-1}$ and $k_{X}^{2 n-1}$ are $H$-maps then $F_{2 n}$ and $X_{2 n}$ are H-spaces and the maps $\pi_{2 n}$ and $k_{F}^{2 n-1}$ are H-maps. 
Lemma 2.2. If $F_{2 n}$ and $X_{2 n}$ are H-spaces and the maps $\pi_{2 n}, k_{X}^{2 n}$ and $k_{F}^{2 n}$ are $H$-maps then $F_{2 n+1}$ and $X_{2 n+1}$ are $H$-spaces and the map $\pi_{2 n+1}$ is an H-map. (Note that (1.6) commutes so that the maps $k_{X}^{2 n}$ and $k_{F}^{2 n}$ are compatible H-maps.)

The proofs of these leınmas are a direct application of Theorem 2 of [2] in diagrams (1.6) and (1.7).

Since in the stable range Lemmas 2.1 and 2.2 hold, the se two lemmas can then be used inductively to get

Proposition 2.3. Let $f: X \rightarrow Y$ satisfy condition $O b l$ and let $X$ be an H-space. If $k_{F}^{2 n}: F_{2 n} \rightarrow K\left(\right.$ coker $\left.; f_{n+1 *}, n+1\right)$ is an H-map for all $n$ then $F$ is an $H$-space and the map $\pi: F \rightarrow X$ is an H-map.

The obstruction to

$$
k_{F}^{2 n}: F_{2 n} \rightarrow K\left(\text { coker } ; f_{n+1 *}, n+1\right)
$$

being an $H$-map lies in $H^{n+1}\left(F_{2 n} \wedge F_{2 n}\right.$; coker $\left.f_{n+1 *}\right)$.

If we consider the diagram

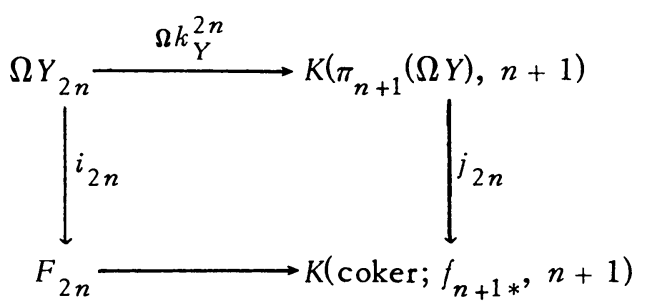

induced from Example 1.3 we have that $k_{F}^{2 n} \circ i_{2 n}=j_{2 n} \circ \Omega k_{Y}^{2 n}$ and that $i_{2 n}$, $\Omega k_{Y}^{2 n}$ and $j_{2 n}$ are all $H$-maps.

Therefore, if we let $\sigma(H) \in H^{n+1}\left(F_{2 n} \wedge F_{2 n}\right.$; coker $\left.f_{n+1 *}\right)$ be the obstruction to $k_{F}^{2 n}$ being an $H$-map we get $\left(i_{2 n} \wedge i_{2 n}\right)^{*}(\sigma(H))$ is zero in $H^{n+1}\left(\Omega Y_{2 n} \wedge \Omega Y_{2 n}\right.$; coker $\left.f_{n+1 *}\right)$.

In particular we get

Proposition 2.4. Let $f: X \rightarrow Y$ satisfy condition $O b l$ and let $X$ be an H-space. If the map

$\left(i_{2 n} \wedge i_{2 n}\right)^{*}: H^{n+1}\left(F_{2 n} \wedge F_{2 n} ;\right.$ coker $\left.f_{n+1 *}\right) \rightarrow H^{n+1}\left(\Omega Y_{2 n} \wedge \Omega Y_{2 n} ;\right.$ coker $\left.f_{n+1 *}\right)$

is monic for all $n$ then $F$ is an H-space and the map $\pi: F \rightarrow X$ is an H-map.

3. External conditions and a proof of Theorem 0.1. Using 2.4 we may prove 
Theorem 3.1. Let $f: X \rightarrow Y$ satisfy Obl. Let $F, \Omega Y$ be simply connected and let $X$ be an H-space. If the map

$$
(i \wedge i)^{*}: H^{n+1}\left(F \wedge F ; \operatorname{coker} f_{n+1 *}\right) \rightarrow H^{n+1}\left(\Omega Y \wedge \Omega Y ; \operatorname{coker} f_{n+1 *}\right)
$$

is monic for each $n$; then $F$ is an H-space and the map $\pi: F \rightarrow X$ is an H-map.

Note that this theorem requires conditions on $f: X \rightarrow Y$ and not on the stages of any $\phi$-Postnikov system. Further note that Theorem 0.1 is an immediate corollary of this.

Proof of Theorem 3.1. By the universal coefficient theorem $H^{n+1}\left(K \wedge K ; \operatorname{coker} f_{n+1 *}\right)$

$$
\simeq \operatorname{Hom}\left(H_{n+1}(K \wedge K) ; \operatorname{coker} f_{n+1 *}\right) \oplus \operatorname{Ext}\left(H_{n}(K \wedge K) ; \operatorname{coker} f_{n+1 *}\right)
$$

for $K=F_{2 n}$ or $\Omega Y_{2 n}$.

By the Künneth formula

$$
H_{m}(K \wedge K) \cong \sum_{p+q=m ; m>p>0} H_{p}(K) \otimes H_{q}(K) \oplus \sum_{p+q=m-1} \operatorname{Tor}\left(H_{p}(K), H_{q}(K)\right) .
$$

But $q_{2 n}^{F}: F \rightarrow F_{2 n}$ and $q_{2 n}^{Y}: \Omega Y \rightarrow \Omega Y_{2 n}$ are both $n-1$ equivalences so that letting $\dot{K}=F_{2 n}$ (or $\Omega Y_{2 n}$ ) and $L=F$ (or $\Omega Y$ ) we get

$$
\begin{aligned}
H_{n+1}(K \wedge K) \cong & \left(H_{n}(K) \otimes H_{1}(K)\right)^{2} \oplus \sum_{p+q=n+1} H_{p}(L) \otimes H_{q}(L) \\
& \oplus \sum_{p+q=n} \operatorname{Tor}\left(H_{p}(L), H_{q}(L)\right) .
\end{aligned}
$$

Since $K$ is simply connected we get that $q: L \rightarrow K$ induces isomorphisms $(q \wedge q)_{*}: H_{i}(L \wedge L) \rightarrow H_{i}(K \wedge K)$ for $i \leq n+1$ and, therefore, we may replace the condition of Proposition 2.4 that the map $\left(i_{2 n} \wedge i_{2 n}\right)^{*}$ be monic with the condition that $(i \wedge i)^{*}$ be monic.

Proof of Theorem 0.2. We will now assume that $H_{*}(Y)$ is free and that $Y$ is $(p-1)$-connected and $F$ is $(q-1)$-connected. From the Serre spectral sequence for the "fibration" $F \rightarrow X \rightarrow Y$ we get that

$$
\cdots \rightarrow H^{i-1}(F) \stackrel{\delta_{F}}{\longrightarrow} H^{i}(Y) \stackrel{f^{*}}{\longrightarrow} H^{i}(X) \stackrel{\pi^{*}}{\longrightarrow} H^{i}(F)
$$

is exact for $i \leq p+q-1$. 
Therefore, if $f^{*}: H^{i}(Y) \rightarrow H^{i}(X)$ is epic for all $i$ we get that $H^{i-1}(\Omega Y)$ $\stackrel{\delta_{Y}}{\rightarrow} H^{i}(Y)$ is an isomorphism for $i \leq 2 p-1$. Thus we get $i^{*}: H^{i}(F) \rightarrow$ $H^{i}(\Omega Y)$ is monic for $i<\min (2 p-1, p+q-1)=l$.

If the dimension of $F$ is less than or equal to $l$ we get $i^{*}: H^{i}(F) \rightarrow$ $H^{i}(\Omega Y)$ is monic for all $i$, and since $H^{i}(Y)$ is free this implies $(i \wedge i)^{*}$ : $H^{i}(F \wedge F) \rightarrow H^{i}(\Omega Y \wedge \Omega Y)$ is monic. Thus Theorem 0.2 is proven by appealing to Theorem 0.1 .

5. Loop spaces. Since $2.1,2.2$, and 2.3 are also trivially true if we replace $H$-space and $H$-map by (the homotopy type of a) loop space and loop map, and since the obstructions to $k_{F}^{2 n}: F_{2 n} \rightarrow K\left(\right.$ coker; $\left.f_{n+1 *}, n+1\right)$ being a loop map are elements

$$
\sigma_{i}(\Omega) \in H^{n+1}\left(\bigwedge^{i}\left(F_{2 n}\right) ; \operatorname{coker} f_{n+1 *}\right) \quad\left(\bigwedge^{i}\left(F_{2 n}\right)=F_{2 n} \wedge \cdots \wedge F_{2 n}\right)
$$

it is easy to see that we have the following result.

Proposition 5.1. Let $f: X \rightarrow Y$ satisfy condition 1.2 and let $X$ be a loop space. If the maps

$$
\left(\bigwedge^{j} i_{2 n}\right)^{*}: H^{n+1}\left(\bigwedge^{j}\left(F_{2 n}\right) ; \operatorname{coker} f_{n+1 *}\right) \rightarrow H^{n+1}\left(\bigwedge^{j}\left(\Omega Y_{2 n}\right) ; \operatorname{coker} f_{n+1 *}\right)
$$

are monic for all $n$ and all $j \geq 2$, then $f$ is a loop space and the map $\pi$ : $F \rightarrow X$ is a loop map.

As in $\S 3$, if we take $F$ and $\Omega Y$ we get a result analogous to Theorem 3.1 but we need the condition $\left(\bigwedge^{i} i\right)^{*}$ is monic for all $n$ and $j \geq 2$, a condition too cumbersome to be of much value. By adding the condition, $H^{*}(Y)$ is free we only need $i_{2 n}$ monic, and hence we get Theorem 0.4 . By assuming that $\pi_{q}(X) \rightarrow \pi_{q}(Y)$ is onto, we get coker $f_{n *}=0$, and hence Proposition 5.1 implies Theorem 0.3 .

\section{REFERENCES}

1. D. W. Kahn, Induced maps for Postnikov systems, Trans. Amer. Math. Soc. 107 (1963), 432-450.

2. J. Stasheff, On extensions of H-spaces, Trans. Amer. Math. Soc. 105 (1962), 126-135. MR $31 \# 2726$.

3. - H-spaces from a homotopy point of view, Lecture Notes in Math., vol. 161, Springer-Verlag, Berlin and New York, 1970. MR 42 \#5261.

4. E. Thomas, Seminar on fibre spaces, Lecture Notes in Math., vol. 13, Springer-Verlag, Berlin and New York, 1966. MR 34 \#3582.

DEPARTMENT OF MATHEMATICS, UNIVERSITY OF NEW HAMPSHIRE, DURHAM, NEW HAMPSHIRE 03824 